\title{
The Clinical Prognostic Value of the
} Neutrophil-to-Lymphocyte Ratio in Brain Metastases from Non-Small Cell Lung Cancer-
Harboring EGFR Mutations

This article was published in the following Dove Press journal:

Cancer Management and Research

\author{
Hongwei $\mathrm{Li}^{1, *}$ \\ Weili Wang ${ }^{1 * *}$ \\ Xiaotang Yang' \\ Jianhong Lian (iD) ${ }^{2}$ \\ Shuangping Zhang ${ }^{3}$ \\ Jianzhong Cao' \\ Xiaqin Zhang' \\ Xin Song' \\ Sufang Jia' \\ Ruiqi Xue ${ }^{4}$ \\ 'Department of Radiotherapy, Shanxi \\ Medical University, Shanxi Cancer \\ Hospital Affiliated to Shanxi Medical \\ University, Taiyuan, Shanxi, People's \\ Republic of China; ${ }^{2}$ Department of \\ Chemotherapy, Shanxi Medical \\ University, Shanxi Cancer Hospital \\ Affiliated to Shanxi Medical University, \\ Taiyuan, Shanxi, People's Republic of \\ China; ${ }^{3}$ Department of Surgery, Shanxi \\ Medical University, Shanxi Cancer \\ Hospital Affiliated to Shanxi Medical \\ University, Taiyuan, Shanxi, People's \\ Republic of China; ${ }^{4}$ Shanxi Medical \\ University, Shanxi Cancer Hospital \\ Affiliated to Shanxi Medical University, \\ Taiyuan, Shanxi, People's Republic of \\ China \\ *These authors contributed equally to \\ this work
}

Correspondence: Xiaotang Yang Department of Radiology, Shanxi Cancer Hospital Affiliated to Shanxi Medical University, Taiyuan, Shanxi, People's

Republic of China

Tel +86 35I-465II 53

Email yangxt210@I26.com
Purpose: Several studies have explored the correlation between the neutrophil-to-lymphocyte ratio (NLR) and the prognosis of patients with lung cancer. However, little is known about the correlation between the pretreatment NLR and the prognosis of patients with brain metastases from non-small cell lung cancer (NSCLC)-harboring mutations in the epidermal growth factor receptor $(E G F R)$ gene. We sought to evaluate the predictive values in brain metastasis from lung adenocarcinoma with $E G F R$ mutations.

Methods: We retrospectively examined 133 patients with brain metastases (BMs) from lung adenocarcinoma with EGFR mutations. NLR was calculated using N/L, where N and L, respectively, refer to peripheral blood neutrophil $(\mathrm{N})$ and lymphocyte $(\mathrm{L})$ counts. The cut-off value of NLR was assessed by the area under the curve (AUC). The Log rank test and Cox proportional hazard model were used to confirm the impact of NLR and other variables on survival.

Results: An NLR value equal to or less than 2.99 was associated with prolonged survival in this cohort of patients in both variable and multivariable analysis.

Conclusion: We concluded that NLR is an independent prognostic factor in BMs from lung adenocarcinoma with EGFR mutations. This could serve as a useful prognostic biomarker and could be incorporated in the clinical prognostic index specific to patients with BMs.

Keywords: neutrophil-to-lymphocyte ratio, brain metastases, non-small cell lung cancer, EGFR mutations

\section{Introduction}

Brain metastases (BMs) are among the most dismal late complications of non-small cell lung cancer (NSCLC). ${ }^{1}$ With standard treatment - that is, whole brain radiation therapy (WBRT) — patients with multifocal metastases has a predicted survival of 4-6 months. Those with oligometastases treated with stereotactic radiosurgery and/or fractionated stereotactic radiotherapy (SRS/SRT) has a predicted survival of 12 months or more. $^{2-4}$ In the past decade, the development of oncologic-targeted therapy has greatly advanced the treatment of NSCLC. Brain metastases with the EGFR mutation treated with tyrosine kinase inhibitors (TKIs) achieved response rates as high as $70 \%$ to $89 \%$ and median overall survival times of 12.9 to 19.8 months compared with the traditional modality with survival times of 6 to 12 months. ${ }^{5-7}$ It has been suggested by most of the guidelines that a TKI, or TKI combined with radiotherapy, should be the first-line treatment option for patients with BMs. ${ }^{8,9}$ 
Systemic inflammation, which promotes tumor progression, is now recognized as an important prognostic factor. Since it can easily be determined from blood tests, many previous studies have demonstrated that elevated inflammatory factors such as the neutrophil-to-lymphocyte ratio (NLR) and platelet-to-lymphocyte ratio (PLR) are associated with poor prognosis in many tumors as well as being related to the sensitivities of chemotherapy, radiotherapy. ${ }^{10-12}$ The prognostic value of systemic inflammation factors was also observed in the management of lung cancer with chemotherapy, radiotherapy, target therapy and immunotherapy. ${ }^{13-17}$ However, there are no clear prognostic factors for patients with BMs from NSCLC with status of EGFR mutation who received target therapy or other treatment modalities. Little is known about the correlation between pretreatment NLR and prognoses of brain metastases. In this study, we aimed to investigate the prognostic value of NLR for response to target therapy or other treatment modalities among brain metastases from NSCLC-harboring EGFR mutation.

\section{Patients and Methods Patients' Eligibility}

Between August 2010 and June 2015 we identified 133 consecutive patients in our cancer center's data base with NSCLC confirmed by histology and with BMs confirmed by computed tomography (CT) and/or magnetic resonance imaging (MRI). All patients were found to have $E G F R$ mutations by DNA direct sequencing or the amplification of refractory mutation systems. Patients who met the following criteria were excluded:

1. History of other malignant tumors

2. Incomplete record of blood test results before BM diagnosis

3. Previous steroid treatment (before blood testing)

4. Less than a 3-month follow-up after the BM diagnosis

This study was approved by the Shanxi Provincial Cancer Hospital ethics committee and conducted in accordance with the Declaration of Helsinki. Written informed consent was obtained from each patient.

\section{Variables}

Each patient's record included the following: gender, age, smoking history, Karnofsky performance score (KPS), control of primary tumor, extent of extracranial disease, number of brain lesions, and graded prognostic assessment (GPA) classes, which were recorded based on the radiation therapy oncology (RTOG) classifications.

The NLRs were calculated using the following equation:

$N L R=N / L$,

where $\mathrm{N}$ and $\mathrm{L}$ refer to peripheral blood neutrophil and lymphocyte counts, respectively. Blood counts were collected from routine blood tests within 7 days of the BM diagnosis. Treatment modalities were grouped as follows: 1) radiotherapy (WBRT/SRS/SRT), 2) EGFR-TKI, 3) combination of EGFR-TKI, and radiotherapy.

\section{Statistical Methods}

The Chi-square or Fisher exact test was used to make comparisons between the two groups. The cut-off value was defined by receiver operating characteristic (ROC) curve analyses. The area under the curve (AUC) was used to assess the predictive value. Overall survival (OS) was estimated from the first day of BM diagnosis to death or date of last follow-up using the Kaplan-Meier method. Group analysis was performed using the Log-rank test for univariate analyses and the Cox proportional hazard model for multivariate analyses. A $P$ value below 0.05 was considered significant. All analyses were performed using SPSS statistical software 22.0.

\section{Results}

\section{Patient Characteristics}

Among the 730 NSCLC patients with BM, 133 were found to have EGFR mutations, including $24.3 \%$ (33) with exon 19 deletions and $24.3 \%$ (33) with exon 21-point mutation (L858R). One patient had both exon 19 and exon 21-point mutations. Two patients were found to harbor other rare types of mutations.

Patients' mean age at the time of diagnosis of BM was 56 years. Most of the patients were female $(60.9 \%)$ and were no smokers (68.4\%). At BM diagnosis, $72.2 \%$ of the patients presented with more than one lesion, and $64.4 \%$ had extracranial metastases. Only $28.6 \%$ of the primary tumors were under control. Patients with KPS 90, 70-80, and 60 accounted for $19.5 \%, 66.9 \%$, and $13.5 \%$, respectively, of the total group. According to RTOG recursive partitioning analyses (RPA) classification system, the number of patients in RPA classes 1, 2, and 3 were 6, 115, and 15 , respectively. ${ }^{18}$ Of the $133 \mathrm{BM}$ cases, 75 had received radiotherapy (WBRT/SRS/SRT) and 65 had received 
EGFR-TKI. The other patients received combined treatments of WBRT plus systemic chemotherapy concurrently or subsequently.

The optimal cut-off value of NLR was calculated by ROC analysis. The AUC for OS was 0.563 . Sensitivity and specificity were 56.79 and 65.38 , respectively. The cut-off value for the prediction of OS in this cohort was 2.99 , as presented in Figure 1. Of the 133 patients, 68 (51.1\%) presented with NLR values equal to or less than 2.99; the remaining 65 (48.9\%) patients had NLR values above 2.99. Between patients with NLR $>2.99$ and $<2.99$, there were no significant differences in terms of clinicopathologic characteristics (Table 1).

\section{Univariate and Multivariate Cox Regression Analysis for OS}

By the end of the follow-up, there were 26 patients alive. The median OS for the whole group was 16.23 months (range: $95 \% \mathrm{CI}=14.03-18.43$ months). In univariate tests, log-rank analysis revealed that age $(P<0.001)$, smoking status $(P=$ $0.037)$, KPS at BM $(P<0.001)$, number of brain lesions $(P=$ $0.029)$ extent of extracranial disease $(P=0.038)$, treatment modality $(P<0.001)$, and NLR values $(P<0.001)$ affected OS. In comparison with the patients with NLR, those with an

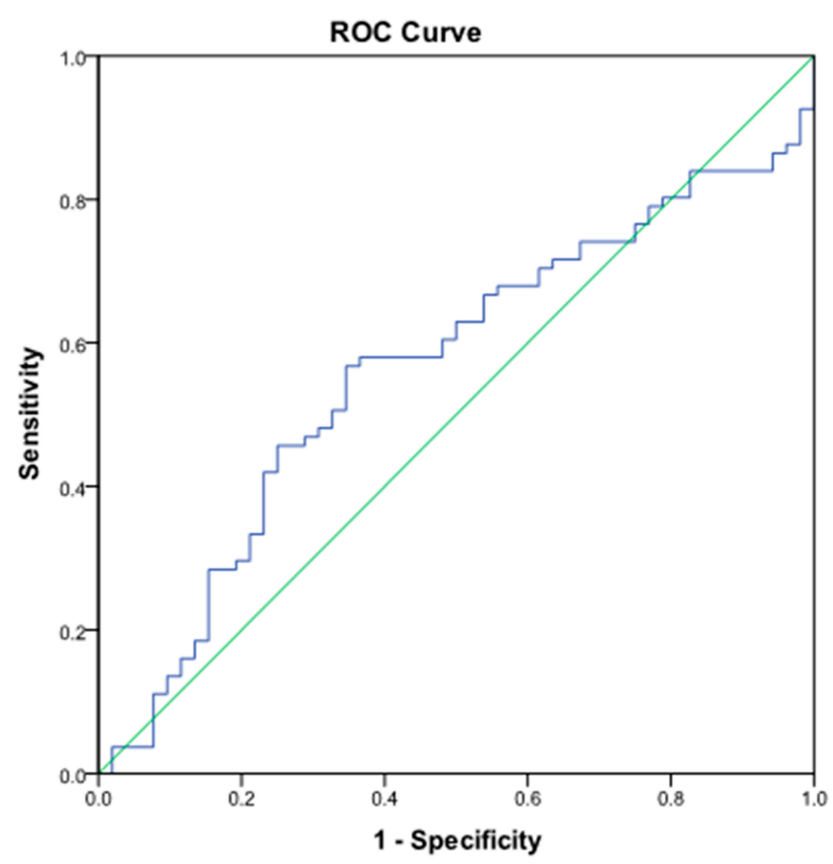

Figure I Receiver operating characteristic curve of the neutrophil-to-lymphocyte ratio. There were 133 patients with epidermal growth factor receptor (EGFR) mutations. The area under the curve is 0.563 and the optimal cut-off value is 2.99. When the optimal cut-off value is chosen, the sensitivity is 56.79 and the specificity is 65.38 .
Table I Characteristics of Patients and the Chi-Square Test

\begin{tabular}{|c|c|c|c|}
\hline \multirow[t]{2}{*}{ Characteristics } & \multirow[t]{2}{*}{ No. (\%) } & \multicolumn{2}{|l|}{$X^{2}$} \\
\hline & & NLR $<2.99 \geq 2.99$ & $\mathbf{P}$ \\
\hline Total & 133 & 6865 & \\
\hline $\begin{array}{l}\text { Gender } \\
\text { Male } \\
\text { Female }\end{array}$ & $\begin{array}{l}52(39.1) \\
81(60.9)\end{array}$ & $\begin{array}{l}2428 \\
4437\end{array}$ & 0.358 \\
\hline $\begin{array}{l}\text { Age } \\
\qquad \begin{array}{l}\leq 65 \\
>65\end{array}\end{array}$ & $\begin{array}{l}109(82) \\
24(18)\end{array}$ & $\begin{array}{l}5356 \\
159\end{array}$ & 0.218 \\
\hline $\begin{array}{l}\text { Smoking } \\
\text { No } \\
\text { Yes }\end{array}$ & $\begin{array}{l}91(68.4) \\
42(31.6)\end{array}$ & $\begin{array}{l}4645 \\
2220\end{array}$ & 0.844 \\
\hline $\begin{array}{l}\text { KPS } \\
\begin{array}{l}\leq 60 \\
70-80 \\
\geq 90\end{array}\end{array}$ & $\begin{array}{l}26(19.5) \\
89(66.9) \\
18(13.5)\end{array}$ & $\begin{array}{l}1016 \\
4742 \\
117\end{array}$ & 0.288 \\
\hline $\begin{array}{l}\text { No. of BMs } \\
\quad \begin{array}{l}1 \\
>1\end{array}\end{array}$ & $\begin{array}{l}37(27.8) \\
96(72.2)\end{array}$ & $\begin{array}{l}1720 \\
5145\end{array}$ & 0.458 \\
\hline $\begin{array}{l}\text { Extracranial metastases } \\
\text { Absent } \\
\text { Present }\end{array}$ & $\begin{array}{l}47(35.3) \\
86(64.7)\end{array}$ & $\begin{array}{l}2126 \\
4739\end{array}$ & 0.272 \\
\hline $\begin{array}{l}\text { TKI treatment } \\
\text { No } \\
\text { Yes }\end{array}$ & $\begin{array}{l}65(48.9) \\
68(51.1)\end{array}$ & $\begin{array}{l}2936 \\
3929\end{array}$ & 0.142 \\
\hline $\begin{array}{l}\text { WBRT } \\
\text { No } \\
\text { Yes }\end{array}$ & $\begin{array}{l}75(56.4) \\
58(43.6)\end{array}$ & $\begin{array}{l}3936 \\
2929\end{array}$ & 0.819 \\
\hline $\begin{array}{l}\text { Primary lesion } \\
\text { Uncontrolled } \\
\text { Controlled }\end{array}$ & $\begin{array}{l}95 \text { (7I.4) } \\
38(28.6)\end{array}$ & $\begin{array}{ll}48 & 47 \\
20 & 18\end{array}$ & 0.826 \\
\hline $\begin{array}{l}\text { NLR } \\
\quad<2.99 \\
\geq 2.99\end{array}$ & $\begin{array}{l}68(51.1) \\
65(48.9)\end{array}$ & - & - \\
\hline
\end{tabular}

Abbreviations: BM, brain metastases; KPS, Karnofsky Performance Status score; WBRT, whole brain radiotherapy; NLR, neutrophil-to-lymphocyte ratio; EGFR, epidermal growth factor receptor; TKI, tyrosine kinase inhibitor. Percentages are in parentheses.

NLR less than 2.99 had a longer OS (20.67 vs 11.43 months). In the subgroup analysis, the results demonstrated that there were significant differences between subgroups by GPA score $(P<0.001)$ (Figure 2). To further determine the independent factors predictive of OS, all of the variables in univariate analysis were entered into a Cox regression model. The results showed that KPS at BM $(\mathrm{HR}=1.805$; $95 \%$ CI: $1.277-2.552$; factors $=0.001$ ), extent of extracranial 


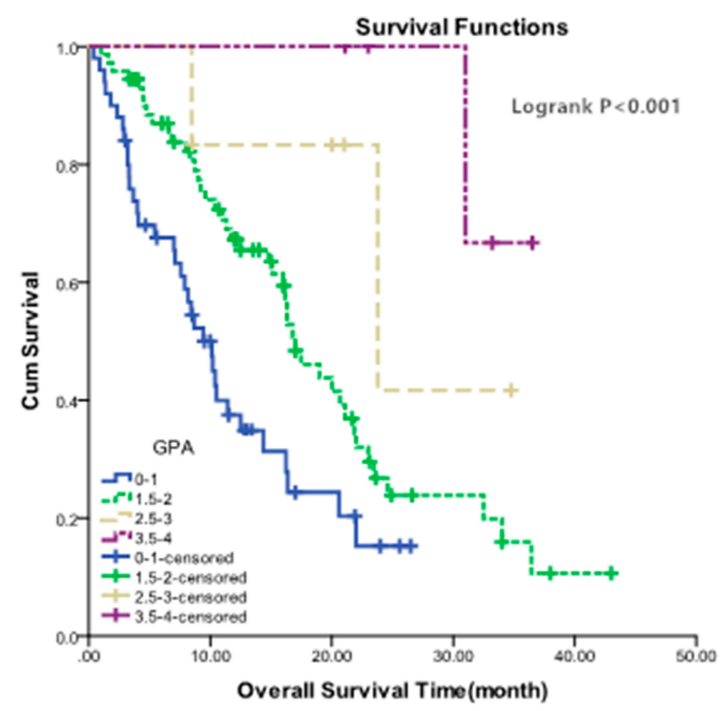

Figure 2 Kaplan-Meier survival curves showing different graded prognostic assessment groups.

disease $(\mathrm{HR}=1.805 ; 95 \% \mathrm{CI}: 1.277-2.552$; factors $=001)$, treatment by $\mathrm{TKI}(\mathrm{HR}=1.805 ; 95 \% \mathrm{CI}: 1.277-2.552$; factors $=0.001)$, and NLR (HR $=1.805 ; 95 \% \mathrm{CI}: 1.277-2.552$; factors $=0.001$ ) were associated with longer OS (Table 2, Figure 3)

\section{Discussion}

The fact that inflammation promotes tumor grown is now widely recognized and inflammation plays an important role in the development and progression of cancer. ${ }^{19}$ In this study, we found that pretreatment of decreased NLR, an important marker of systemic inflammation, was associated with better OS in patients with brain metastases from lung adenocarcinoma and EGFR mutations.

Neutrophils are a type of innate immune cells that are involved in the clearance of microbial infection. They are recruited into the microenvironment of tumors depending on chemokines that bind to CXCR1 and CXCR2 which are expressed by neutrophils. ${ }^{20}$ The changed microenvironment can then accelerate tumor growth, invasion, and metastasis. Studies have confirmed that increases in the number of neutrophils are associated with poor prognoses in NSCLC. ${ }^{21}$ As an important part of the immune system, lymphocytes play a key role in the immune response to cancer. A low lymphocyte count indicates immunosuppressing status inside the body. Lymphopenia has been demonstrated to be associated with a poor prognosis in many types of cancer. $^{22}$ NLR represents the balance between neutrophils and lymphocytes. An elevated NLR indicates decreasing antitumor effects of T-lymphocytes and the releasing of inflammatory cytokines by neutrophils, thus promoting tumor growth, invasion, and metastasis by stimulating the tumor microenvironment. Since 1990, when Letomi first proposed that NLR may be associated with the prognosis of tumors, many studies have suggested that an elevated NLR may be an independent negative prognostic factor in a wide variety of cancers treated by different modalities. ${ }^{23-26}$ A recent meta-analysis, including 14 studies with 2734 lung cancer patients, showed that an elevated NLR can predict a worse OS from multivariate or univariate analysis. Subgroup multivariate analysis showed that a high NLR yielded a worse OS in 2433 NSCLC patients. ${ }^{27}$ This implied that NLR could serve as a biologic maker predicting long-term survival in patients with NSCLC.

In recent decades, the gene underlying EGFR mutations was found to be a biological marker of NSCLC. It is now widely accepted that the response to EGFR TKI is greater in patients with tumor-harboring $E G F R$ mutations than in patients without $E G F R$ mutations. For patients with brain metastases from lung adenocarcinoma identified with EGFR mutations, EGFR TKIs also showed high efficacy in brain lesions. Targeted therapy alone or in combination with radiotherapy was recognized as the first-line treatment modality in this group of patients. 8,9

Although technologic advances have led to improved outcomes for this disease, the OS rates remain disappointingly low, ranging from 12.9 to 18.8 months. ${ }^{5-7}$ Attempts should be made to identify factors other than disease stage and EGFR mutation to serve as tools for selecting proper treatment approaches. In one large pooled study of BMs, age, gender, number of brain lesions, control of primary tumors, and the presence of extracranial metastases were identified as important prognostic factors for survival in patients with BMs from different cancers. ${ }^{28}$ However, for this specific subgroup of NSCLC BM patients with EGFR mutations, besides radiotherapy, EGFR-TKI was included in the treatment strategy. Newer and more reliable markers are needed to identify those patients most likely to benefit from treatment. In our study, we analyzed 133 NSCLC BM patients identified with EGFR mutations. The results showed that pretreatment and treatment factors including age below 65, nonsmoker, high KPS, lower number of brain lesions, absence of extracranial metastases, and being treated with WBRT or EGFR-TKI were associated with a longer overall survival. This is consistent with many previous studies. We also employed a new factor NLR as a prognostic factor. Both in univariate and 
Table 2 Univariate and Multivariate Analyses

\begin{tabular}{|c|c|c|c|c|c|c|c|}
\hline \multirow[t]{2}{*}{ Characteristics } & \multicolumn{4}{|l|}{ Log-Rank } & \multicolumn{3}{|l|}{$\operatorname{cox}$} \\
\hline & MST (m) & SE & $95 \% \mathrm{Cl}$ & $\mathbf{P}$ & HR & $95 \% \mathrm{Cl}$ & $\mathbf{P}$ \\
\hline \multicolumn{8}{|l|}{ Gender } \\
\hline Male & 16.23 & 2.427 & I I.473-20.987 & 0.897 & 1.761 & $0.912-3.399$ & 0.092 \\
\hline Female & 16.30 & 1.297 & $13.758-18.842$ & & & & \\
\hline \multicolumn{8}{|l|}{ Age } \\
\hline$\leq 65$ & 16.77 & 1.486 & $13.857-19.683$ & 0.064 & 2.95 & $1.654-5.260$ & $<0.0$ \\
\hline$>65$ & 10.40 & 2.423 & $5.652-15.148$ & & 0 & & 01 \\
\hline \multicolumn{8}{|l|}{ Smoking } \\
\hline No & 16.30 & 2.119 & $12.147-20.453$ & 0.837 & 2.13 & $|.045-4.37|$ & 0.037 \\
\hline Yes & 16.00 & 3.998 & $8.165-23.835$ & & 8 & & \\
\hline \multicolumn{8}{|l|}{ KPS } \\
\hline$\leq 60$ & 3.33 & 1.055 & $1.262-5.398$ & $<0.001$ & 0.066 & $0.027-0.160$ & $<0.001$ \\
\hline $70-80$ & 16.23 & 0.666 & $14.924-17.536$ & & & & \\
\hline$\geq 90$ & 24.60 & 7.188 & $10.512-38.688$ & & & & \\
\hline No. of BMs & & & & & & & 0.029 \\
\hline 1 & 19.00 & 3.254 & $12.622-25.378$ & 0.128 & 1.827 & $1.063-3.139$ & \\
\hline$>2$ & 14.37 & 2.134 & $10.188-18.552$ & & & & \\
\hline Extracranial metastases & & & & & & & 0.002 \\
\hline Absent & 24.60 & 7.312 & $|0.269-38.93|$ & 0.015 & 2.464 & $1.400-4.339$ & \\
\hline Present & 14.37 & 2.704 & $9.070-19.670$ & & & & \\
\hline \multicolumn{8}{|l|}{ TKI treatment } \\
\hline No & 11.30 & 1.356 & $8.642-13.958$ & 0.005 & 0.516 & $0.314-0.846$ & 0.009 \\
\hline Yes & 17.47 & 2.136 & |3.283-21.657 & & & & \\
\hline WBRT & & & & & & & 0.043 \\
\hline No & 20.00 & 2.431 & $15.236-24.764$ & 0.147 & 1.660 & $1.016-2.712$ & \\
\hline Yes & 12.50 & 2.628 & $7.350-17.650$ & & & & \\
\hline \multicolumn{8}{|l|}{ Primary lesion } \\
\hline Uncontrolled & 16.00 & 2.180 & | I. .728-20.272 & 0.561 & 0.809 & $0.461-1.420$ & 0.460 \\
\hline Controlled & 17.00 & 2.611 & $11.883-22.117$ & & & & \\
\hline \multicolumn{8}{|l|}{ NLR } \\
\hline$<2.99$ & 20.67 & 2.559 & $15.655-25.685$ & 0.004 & 2.122 & $1.276-3.529$ & 0.004 \\
\hline$\geq 2.99$ & 11.43 & 1.000 & $9.470-13.390$ & & & & \\
\hline
\end{tabular}

Abbreviations: BM, brain metastases; KPS, Karnofsky Performance Status score; WBRT, whole brain radiotherapy; NLR, neutrophil-to-lymphocyte ratio; EGFR, epidermal growth factor receptor; TKI, tyrosine kinase inhibitor; MST, median survival time; SE, standard error; Cl, confidence Interval; HR, hazard ratio.

multivariate tests, elevated NLR was a significant prognostic factor in this cohort of patients. Several previous studies reported that low NLR values were strongly correlated with better PFS and OS in EGFR mutated NSCLC patients receiving EGFR-TKIs. ${ }^{16,29}$ The present study focus on specific cohort of EGFR mutated NSCLC patients with BMs. It indicates that inflammatory factors such as NLR could serve as new biologic markers in the management of this group of patients with BMs.

To assess patients' prognoses and identify the best treatment options, several prognostic indices are used and others are being developed. These are based mainly on data from
BM patients who are receiving radiotherapy. ${ }^{18,28,30}$ In the present study, we also categorized patients according to an RTOG recursive partitioning analysis (RPA) classification system. The results showed that there are significant differences among subgroups. The analysis confirmed that this prognostic index may be appropriate for BM patients receiving targeted therapy. However, according to our data, other independent prognostic factors, such as pretreatment NLR, should be incorporated within future systems.

There are several limitations in this study. First, the treatment modalities among this cohort of patients varied. Owing 


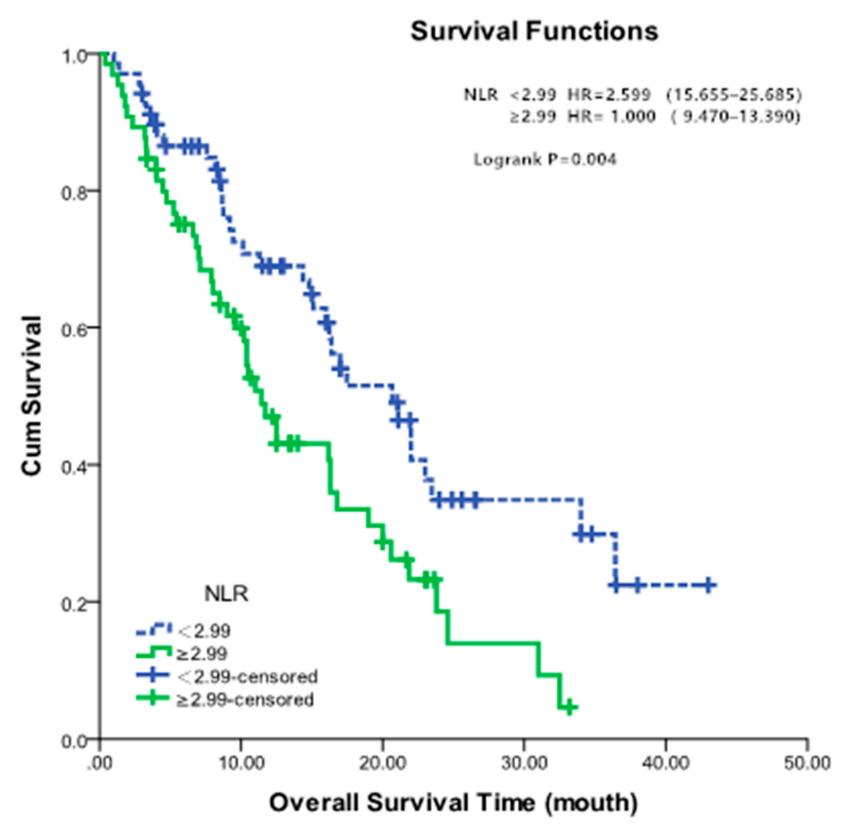

Figure 3 Kaplan-Meier survival curves showing different neutrophil-to-leukocyte ratio groups. NLR less than 2.99 had a longer OS (20.67 vs II.43 months).

to our relatively small sample size, subgroup analysis by treatment modalities was not possible. Second, our cut-off NLR value was 2.99. However, the literature offers no widely accepted cut-off value for NLR. Earlier lung cancer studies reported values ranging from 2.5 to $5 .^{27}$ This might decrease the applicability of NLR as a biologic prognostic factor in routine clinical practice. To define a standard value, future large, multi-center, studies are needed. Third, because the data were collected from a single medical center and are retrospective in nature, some inconsistencies that may have influenced our results were inevitable.

In conclusion, elevated NLR was a poor predictor of survival in brain metastases from NSCLC with EGFR mutations. The NLR may serve as a useful prognostic biomarker and should be incorporated in the clinical prognostic index for these kinds of patients. However, the utility of the NLR remains to be confirmed in future analyses.

\section{Acknowledgments}

We thank LetPub for its linguistic assistance during the preparation of this manuscript. The authors also thank Songyun Han for date preparation and suggestions for revision of the manuscript.

\section{Funding}

Natural Science Foundation of Shanxi Province in China (201701D12116).

\section{Disclosure}

The authors report no conflicts of interest in this work.

\section{References}

1. Bray F, Ren JS, Masuyer E, Ferlay J. Global estimates of cancer prevalence for 27 sites in the adult population in 2008. Int $J$ Cancer. 2013;132:1133-1145. doi:10.1002/ijc.27711

2. Gaspar LE, Mehta MP, Patchell RA, et al. The role of whole brain radiation therapy in the management of newly diagnosed brain metastases: a systematic review and evidence-based clinical practice guideline. J Neurooncol. 2010;96:17-32. doi:10.1007/s11060-009-0060-9

3. Patil CG, Pricola K, Sarmiento JM, Garg SK, Bryant A, Black KL. Whole brain radiation therapy (WBRT) alone versus WBRT and radiosurgery for the treatment of brain metastases. Cochrane Database Syst Rev. 2012;12:CD006121.

4. Yamamoto M, Serizawa T, Shuto T, et al. Stereotactic radiosurgery for patients with multiple brain metastases (JLGK0901): a multiinstitutional prospective observational study. Lancet Oncol. 2014;15 (4):387-395. doi:10.1016/S1470-2045(14)70061-0

5. Li Z, Guo H. The retrospective analysis of the frequency of EGFR mutations and efficacy of gefitinib in NSCLC patients with brain metastases. J Clin Oncol. 2011;29:e18065. doi:10.1200/jco.2011. 29.15 suppl.e1 18065

6. Wu C, Li YL, Wang ZM, Li Z, Zhang TX, Wei Z. Gefitinib as palliative therapy for lung adenocarcinoma metastatic to the brain. Lung Cancer. 2007;57:359-364. doi:10.1016/j.lungcan.2007.03.011

7. Porta R, Sánchez-Torres JM, Paz-Ares L, et al. Brain metastases from lung cancer responding to erlotinib: the importance of EGFR mutation. Eur Respir J. 2011;37(3):624-631. doi:10.1183/09031936. 00195609

8. National Comprehensive Cancer Network(NCCN). Clinical Practice Guidelines in Oncology: non-Small Cell Lung Cancer (Version 2.2019). Available from: https://www.nccn.org/professionals/physi cian_gls/f_guidelines.asp. Accessed July 1, 2020.

9. Novello S, Barlesi F, Califano R, et al. ESMO guidelines committee metastatic non-small-cell lung cancer: ESMO clinical practice guidelines for diagnosis, treatment and follow-up . Ann Oncol. 2016;27: v1e27.

10. Zhou Y, Wei Q, Fan J, Cheng S, Ding W, Hua Z. Prognostic role of the neutrophil-to-lymphocyte ratio in pancreatic cancer: a meta analysis containing 8252 patients. Clin Chim Acta. 2018;479:181-189. doi:10.1016/j.cca.2018.01.024

11. Yang JJ, Hu ZG, Shi WX, Deng T, He SQ, Yuan SG. Prognostic significance of neutrophil to lymphocyte ratio in pancreatic cancer: a meta-analysis. World $J$ Gastroenterol. 2015;21:2807-2815. doi:10.3748/wjg.v21.i9.2807

12. Feng F, Tian Y, Liu S, et al. Combination of PLR, MLR, MWR, and tumor size could significantly increase the prognostic value for gastrointestinal stromal tumors. Medicines. 2016;95:e3248.

13. Yuan C, Li N, Mao X, Liu Z, Ou W, Wang SY. Elevated pretreatment neutrophil/white blood cell ratio and monocyte/lymphocyte ratio predict poor survival in patients with curatively resected non-small cell lung cancer: results from a large cohort. Thorac Cancer. 2017;8 (4):350-358. doi:10.1111/1759-7714.12454

14. Scilla KA, Bentzen SM, Lam VK, et al. Neutrophil-lymphocyte ratio is a prognostic marker in patients with locally advanced (Stage IIIA and IIIB) non-small cell lung cancer treated with combined modality therapy. Oncologist. 2017;22(6):737-742. doi:10.1634/theoncologist.2016-0443

15. Bremnes RM, Al-Shibli K, Donnem T, et al. The role of tumorinfiltrating immune cells and chronic inflammation at the tumor site on cancer development, progression, and prognosis: emphasis on non-small cell lung cancer. J Thorac Oncol. 2011;6:824-833. doi:10.1097/JTO.0b013e3182037b76 
16. Zhang Y, Feng Y-C, Zhu H-G, et al. The peripheral blood neutrophilto-lymphocyte ratio is a prognostic predictor for survival of EGFRmutant non small cell lung cancer patients treated with EGFR-TKIs. Medicine (Baltimore). 2018;97(30):e11648.

17. Russo A, Russano M, Franchina T, AL ET. Neutrophil-toLymphocyte Ratio (NLR), Platelet-to-Lymphocyte Ratio (PLR), and Outcomes with Nivolumab in Pretreated Non-Small Cell Lung Cancer (NSCLC): a large retrospective multicenter study. Adv Ther. 2020;37(3):1145-1155. doi:10.1007/s12325-020-01229-w

18. Sperduto PW, Chao ST, Sneed PK, et al. Diagnosis specific prognostic factors, indexes, and treatment outcomes for patients with newly diagnosed brain metastases: a multi-institutional analysis of 4,259 patients. Int $J$ Radiat Oncol Biol Phys. 2010;77:655-661. doi:10.1016/j.ijrobp.2009.08.025

19. Grivennikov SI, Greten FR, Karin M. Immunity, inflammation, and cancer. Cell. 2010;140:883-899. doi:10.1016/j.cell.2010.01.025

20. Tazzyman S, Barry ST, Ashton S, et al. Inhibition of neutrophil infiltration into A549 lung tumors in vitro and in vivo using a CXCR2-specific antagonist is associated with reduced tumor growth. Int J Cancer. 2011;129:847-858. doi:10.1002/ijc.25987

21. Teramukai S, Kitano T, Kishida Y, Kawahara M, Kubota K. Pretreatment neutrophil count as an independent prognostic factor in advanced non-small-cell lung cancer: an analysis of Japan Multinational Trial Organisation LC00-03. Eur J Cancer. 2009;45 (11):1950-1958. doi:10.1016/j.ejca.2009.01.023

22. von Bernstorff W, Voss M, Freichel S, et al. Systemic and local immunosuppression in pancreatic cancer patients. Clin Cancer Res. 2001;7(3 Suppl):925s-932s.

23. Ietomi K. A study on the role of granulocytes in carcinoma-bearing hosts-G/L ratio as a new host indicator. Nihon Gan Chiryo Gakkai Shi. 1990;25(3):662-671.
24. Cao J, Zhu X, Zhao X, Xue-Feng L, Xu R. Neutrophil-to-lymphocyte ratio predicts PSA response and prognosis in prostate cancer: a systematic review and meta-analysis. PLoS One. 2016;11(7): e0158770. doi:10.1371/journal.pone.0158770

25. Bowen RC, Nancy Ann B, Little JR, et al. Khong Neutrophil-tolymphocyte ratio as prognostic indicator in gastrointestinal cancers: a systematic review and meta-analysis. Oncotarget. 2017;8(19):3217132189. doi:10.18632/oncotarget.16291

26. Najjar M, Agrawal S, Emond JC, Halazun KJ. Pretreatment neutrophil-lymphocyte ratio: useful prognostic biomarker in hepatocellular carcinoma. J Hepatocell Carcinoma. 2018;5:17-28.

27. Yin Y, Jun Wang I II, Wang X, et al. Prognostic value of the neutrophil to lymphocyte ratio in lung cancer: a meta-analysis. CLINICS. 2015;70(7):524-530. doi:10.6061/clinics/2015(07)10

28. Gaspar L, Scott C, Rotman M, et al. Recursive partitioning analysis (RPA) of prognostic factors in three Radiation Therapy Oncology Group (RTOG) brain metastases trials. Int J Radiat Oncol Biol Phys. 1997;37(4):745-751. doi:10.1016/S0360-3016(96)00619-0

29. Chen YM, Lai CH, Chang HC, et al. Baseline and trend of lymphocyte-to- monocyte ratio as prognostic factors in epidermal growth factor receptor mutant non-small cell lung cancer patients treated with first-line epidermal growth factor receptor tyrosine kinase inhibitors. PLoS One. 2015;10:e136252.

30. Lorenzoni J, Devriendt D, Massager N, et al. Radiosurgery for treatment of brain metastases: estimation of patient eligibility using three stratification systems. Int J Radiat Oncol Biol Phys. 2004;60(1):218224. doi:10.1016/j.ijrobp.2004.02.017

\section{Publish your work in this journal}

Cancer Management and Research is an international, peer-reviewed open access journal focusing on cancer research and the optimal use of preventative and integrated treatment interventions to achieve improved outcomes, enhanced survival and quality of life for the cancer patient.
The manuscript management system is completely online and includes a very quick and fair peer-review system, which is all easy to use. Visit http://www.dovepress.com/testimonials.php to read real quotes from published authors. 